\title{
EpCAM as multi-tumour target for near- infrared fluorescence guided surgery
}

P. B. A. A. van Driel ${ }^{1,2 \dagger}$, M. C. Boonstra ${ }^{4 \dagger}$, H. A. J. M. Prevoo ${ }^{4}$, M. van de Giessen ${ }^{3}$, T. J. A. Snoeks ${ }^{1}$,

Q. R. J. G. Tummers ${ }^{4}$, S. Keereweer ${ }^{5}$, R. A. Cordfunke ${ }^{6}$, A. Fish'7, J. D. H. van Eendenburg ${ }^{8}$, B. P. F. Lelieveldt ${ }^{3}$, J. Dijkstra ${ }^{3}$, C. J. H. van de Velde ${ }^{4}$, P. J. K. Kuppen ${ }^{4,9}$, A. L. Vahrmeijer ${ }^{4}$, C. W. G. M. Löwik ${ }^{1}$ and C. F. M. Sier , $^{4 *}$

\begin{abstract}
Background: Evaluation of resection margins during cancer surgery can be challenging, often resulting in incomplete tumour removal. Fluorescence-guided surgery (FGS) aims to aid the surgeon to visualize tumours and resection margins during surgery. FGS relies on a clinically applicable imaging system in combination with a specific tumour-targeting contrast agent. In this study EPCAM (epithelial cell adhesion molecule) is evaluated as target for FGS in combination with the novel Artemis imaging system.

Methods: The NIR fluorophore IRDye800CW was conjugated to the well-established EpCAM specific monoclonal antibody 323/A3 and an isotype IgG1 as control. The anti-EpCAM/800CW conjugate was stable in serum and showed preserved binding capacity as evaluated on EPCAM positive and negative cell lines, using flow cytometry and cell-based plate assays. Four clinically relevant orthotopic tumour models, i.e. colorectal cancer, breast cancer, head and neck cancer, and peritonitis carcinomatosa, were used to evaluate the performance of the anti-EpCAM agent with the clinically validated Artemis imaging system. The Pearl Impulse small animal imaging system was used as reference. The specificity of the NIRF signal was confirmed using bioluminescence imaging and green-fluorescent protein.
\end{abstract}

Results: All tumour types could clearly be delineated and resected $72 \mathrm{~h}$ after injection of the imaging agent. Using NIRF imaging millimetre sized tumour nodules were detected that were invisible for the naked eye. Fluorescence microscopy demonstrated the distribution and tumour specificity of the anti-EpCAM agent.

Conclusions: This study shows the potential of an EpCAM specific NIR-fluorescent agent in combination with a clinically validated intraoperative imaging system to visualize various tumours during surgery.

Keywords: Image-guided surgery, Near-infrared fluorescence, Optical imaging, Epithelial cell adhesion molecule, Imaging agent

\section{Background}

Curative intended oncologic surgery aims to completely resect all malignant cells. Discriminating tumour from healthy tissue during surgery is therefore of paramount importance [1]. As a consequence, clinically complete resected tumours frequently turn out to be pathological incompletely removed [2-4]. Therefore, novel intraoperative imaging modalities are needed that aid the

\footnotetext{
*Correspondence: c.f.m.sier@lumc.nl

${ }^{\dagger}$ Equal contributors

${ }^{4}$ Department of Surgery, Leiden University Medical Centre, Leiden, Netherlands

${ }^{9}$ Antibodies for Research Applications BV, Gouda, The Netherlands Full list of author information is available at the end of the article
}

surgeon in recognizing tumour spread and provide guidance during tumour removal. Fluorescence-guided surgery (FGS) is a technique based on near-infrared (NIR) light, which has been widely investigated for sentinel lymph node procedures, anastomosis and during cholecystectomies [5-7]. The advantage of NIR fluorescent light is the relatively deep tissue penetration and the minimal tissue auto fluorescence at these wavelengths. The tissue depth at which a fluorophore can be detected is dependent on the fluorophore, the tissue optical properties and the sensitivity of the imaging device. Maximum tissue penetration detection has been estimated to be between 3 and $4 \mathrm{~cm}$ for intensified devices [8] and up to $2 \mathrm{~cm}$ for 
others [9]. Further, since NIR fluorescence light is invisible for the human eye, there is no alteration of the surgical field [10]. The major challenges for the routine introduction of FGS in the clinic comprise the availability of validated NIR fluorescence imaging systems in combination with a dedicated tumour-specific NIR fluorescence agent [11].

The first NIR imaging systems for clinical application proved the feasibility of the concept but were in fact more proto-types than standard clinical equipment. The next generation NIR imaging systems, as used in this study, are more versatile, smaller, cheaper, and more sensitive and should meet uniform standards warranting an exponential increase for clinical applications. Therefore, presently the biggest challenge for clinical introduction of FGS is the development of specific tumour targeting NIR fluorescent agents that comply with these second generation imagers. Various established membrane-bound tumour markers are under evaluation as targets for (NIR) fluorescence imaging in pre-clinical settings, such as EGFR, HER2/Neu, $\operatorname{VEGF(R),~folate~receptor~alpha,~}$ UPAR and various integrins [12-17]. Although these proteins have been successfully targeted in human tumours xenografted in animal models, none of them seems to be the universal target suited for the majority of tumour (types) in a clinical setting: These proteins are either present on the majority of tumours but only on a low percentage of tumour (stromal) cells, like VEGFR and $\alpha_{v} \beta_{3}$ integrin, or they are abundantly present in only a limited percentage of tumour (types), like folate receptor, EGFR and HER2.

In this study we evaluate Epithelial Cell Adhesion Molecule (EpCAM) as target for FGS. EpCAM is a transmembrane glycoprotein involved in cell-cell interactions and cell-stroma adhesion [18]. EpCAM expression is restricted to epithelial cells and is highly up-regulated in virtually all epithelial carcinomas $[19,20]$. EpCAM upregulation is associated with cancer progression and EpCAM is found on circulating tumour cells and metastases. EpCAM overexpression in cancer cells was found to be 100- to 1000-fold higher compared to expression on normal breast cells resulting in 100,000 to 400,000 copies per cell [21]. Compared to an established tumour target like EGFR and Her2/Neu this number is only marginally less. But overexpression of EGFR and HER2/Neu in primary breast cancer is reported to be $0.8-14$ and $15-20 \%$ respectively, whereas $40-98 \%$ of the primary breast tumours show enhanced EpCAM levels [20, 22]. For other epithelial cancers these figures are similar or favour the use of EpCAM as imaging target even more [20, 23-25]. These findings have led to the development of many EpCAM specific antibodies, from which some have been evaluated in phase I, II and III immunotherapeutic trials in various cancer types, such as ovarian, gastric and head-andneck cancer [26, 27]. The results of these studies did not meet the high expectations for the therapeutic purpose and most of these investigations were aborted. Mild adverse effects like nausea, vomiting and elevation of pancreatic enzymes have been reported for therapeutic use, but are not expected in single dose imaging studies. The potential of EpCAM targeting has recently been re-discovered, as tumour specific imaging was demonstrated for SPECT imaging. A monoclonal antibody with medium high affinity for EpCAM, has been labelled with various radionuclides and has been extensively evaluated in several xenografted tumour models in mice $[28,29]$.

In the current study we conjugated the same monoclonal antibody 323/A3 to the clinically relevant NIR fluorescent dye IRDye $800 \mathrm{CW}$ and evaluated this conjugate in tumour models of clinically relevant orthotopic colorectal, breast and head-and-neck cancer models. To simulate clinical conditions we evaluated the performance of the probe with the recently introduced commercially available Artemis imaging system [30] in comparison with the Pearl Impulse small animal imaging system, a standard apparatus for use in pre-clinical settings.

\section{Methods \\ Cell lines}

For colorectal, breast and head-and-neck cancer, we selected two cell lines with different tumour characteristics. Luciferase transfected cells were used to follow orthotopic tumour growth by bioluminescence imaging (BLI). MCF-7 and OSC-19 cells were transfected with Luciferase 2 and green fluorescent protein as described previously [31]. All cell lines were grown in a humidified incubator at $37{ }^{\circ} \mathrm{C}$ and $5 \% \mathrm{CO}_{2}$. Cells were cultured for not more than 10 passages and regularly checked for Mycoplasma infection by PCR.

\section{EpCAM expression}

EpCAM expression of HT29(-/+)luc2, COLO320, OSC19-luc2-cGFP, FaDu-luc2, MCF-7-luc2-cGFP and MDAMB-231 cells was evaluated by flow cytometry. Cells were cultured until 90\% confluence and detached with trypsin. Viability of the cells was evaluated using trypan blue. After adjusting the number of cells to $0.5 \times 10^{6}$ per tube in ice cold phosphate-buffered saline (PBS), they were incubated with $0.4 \mu \mathrm{g} / \mathrm{ml} 323 / \mathrm{A} 3$ anti-EpCAM antibody or isotype control MOPC21 for $30 \mathrm{~min}$ on ice. Then cells were washed three times in ice cold PBS and incubated with a goat anti-mouse IgG1-AF488 secondary antibody (Invitrogen, $2.5 \mu \mathrm{g} / \mathrm{ml}$ ). The cells were washed three times in ice cold PBS and resuspended in $400 \mu \mathrm{L}$ PBS containing propidium iodide to exclude dead cells from the analysis. Flow cytometry was performed using the LSRII (BD Biosciences). The experiments were performed 
in duplicate and EpCAM expression was estimated as the geometric mean of fluorescence intensity measured in 10,000 viable cells. For quantitative determination of EpCAM numbers per cell type the Qifikit (Dako) was used.

\section{Antibodies and conjugation to IRDye $800 \mathrm{CW}$}

EpCAM specific monoclonal chimeric antibody 323/ A3 and the $\operatorname{IgG}_{1 \mathrm{k}}$ isotype control monoclonal antibody MOPC21 (BioXcell, West Lebanon, USA) were used [32]. Antibody 323/A3 has a medium high affinity $\left(K_{\alpha}=2 \times\right.$ $10^{9} \mathrm{M}^{-1}$ ) for EpCAM and is directed against the EGF-like domain I epitope on the extracellular domain of the EpCAM molecule, whereas MOPC21 has an unknown specificity after testing on human and rodent tissues [33-35]. Both antibodies were covalently conjugated to NIR fluorochrome IRDye 800CW (LI-COR, Lincoln, NE, USA). $\lambda \mathrm{ex}=773 \mathrm{~nm}, \lambda \mathrm{em}=792 \mathrm{~nm}$ ) using $\mathrm{N}$ hydroxysuccinimide ester chemistry as indicated by the manufacturer. Removal of unconjugated fluorophore was accomplished by using two Zeba Spin Desalting columns (Thermo Fisher Scientific, Perbio Science Nederland B.B., Etten-Leur, The Netherlands) per protein in two sequential steps. For comparison experiments, the two conjugates i.e. the EpCAM specific (323/A3-800CW) and control (MOPC21-800CW) were complemented by the chemically inactive carboxylate version of IRDye $800 \mathrm{CW}$, representing the fluorescent label without antibody control.

\section{Serum stability}

The stability of 323/A3-800CW in human serum was evaluated using HPLC (Biosep-SEC-s2000, Phenomenex, USA). Serum and sodium azide dilution were filtrated through a $0.22 \mu \mathrm{m}$ filter in a $15 \mathrm{ml}$ tube. A 24-wells plate (Greiner Bio-one, Germany) was prepared with $0.02 \%$ sodium azide and serum/probe in a ratio of $1: 1$ and PBS as control and incubated at $37{ }^{\circ} \mathrm{C}$ under $5 \% \mathrm{CO}_{2}$. At 4, 24, 48 and $96 \mathrm{~h} 20 \mu \mathrm{l}$ of sample, diluted in $40 \mu \mathrm{L}$ PBS was evaluated using HPLC in PBS at a flow rate of $0,5 \mathrm{ml} / \mathrm{min}$ for $60 \mathrm{~min}$, detected at 2 channels, 280 and $780 \mathrm{~nm}$.

\section{Cell binding study}

A cell binding assay was performed to confirm the EpCAM specificity of 323/A3-800CW. HT29-luc2 (40,000 cells), COLO320 (40,000 cells), OSC-19-luc2-cGFP (25,000 cells), FaDu-luc2 (35,000 cells), MCF-7-luc2-cGFP (40,000 cells) and MDA-MB-231 cells (40,000 cells) cells were seeded in a black 96-well plate (Greiner Bio-one, Germany). At $\pm 90 \%$ confluence the cells were washed twice with PBS. 323/A3-800CW and MOPC21-800CW were added in a concentration range of $0-8 \mu \mathrm{g} / \mathrm{ml}$ and incubated for $1 \mathrm{~h}$ at $37^{\circ} \mathrm{C}$. After incubation, the cells were washed twice with culture medium without supplements. Bound antibody was imaged with an Odyssey scanner
(LI-COR), scanning at the $800 \mathrm{~nm}$ channel. To correct the fluorescence signal for the number of tumour cells per well a cell nucleus staining was performed: The cells were fixed/permeabilized with acetone/methanol for 10 min, washed with PBS, and incubated with TO-PRO-3 (Invitrogen) at 1:1000 for $5 \mathrm{~min}$ at room temperature. After washing twice with PBS, the plate was imaged with the Odyssey scanner at the $700 \mathrm{~nm}$ channel to detect TO-PRO-3 fluorescence. The ratio of the 800 and $700 \mathrm{~nm}$ fluorescence was plotted. The experiments were performed in triplicate.

\section{Animal models}

Nude Balb/c female mice (Charles River laboratories, l'Arbresle, France), aged 4-6 weeks, were housed in individually ventilated cages and provided with food and sterilized water ad libitum. Their general health state was monitored by weight measurements throughout the experiments. Tumour growth was monitored longitudinally by visual inspection of the tumours, caliper measurements and/or by bioluminescence imaging. Bioluminescence imaging was performed by intraperitoneal injecting of $150 \mathrm{mg} / \mathrm{kg}$ of D-luciferin solution (SynChem, Inc, Elk Grove Village, IL) in PBS, in a total volume of $50 \mu \mathrm{L}$. After $10 \mathrm{~min}$, mice were imaged with the IVIS Spectrum imaging system (PerkinElmer, Waltham, MA, USA). Imaging procedures were performed under isoflurane gas anaesthesia

Colon cancer models

To induce colon tumours, mice were subcutaneously injected at four sites with $5 \times 10^{5}$ HT29 cells in $40 \mu \mathrm{L}$ RPMI1640 medium. Tumour growth was followed by calliper measurements and after 10 days, when the tumours reached a volume of approximately $75 \mathrm{~mm}^{3}$, imaging experiments started. Orthotopic HT29-luc2 tumours were induced as described previously [36]. To induce orthotopic breast tumours, $2.5 \times 10^{5} \mathrm{MCF}-7$ luc2-cGFP cells were inoculated in two contralateral mammary fat pads. Oestrogen pellets (17 $\beta$ oestradiol, $0.36 \mathrm{mg} /$ pellet, 60 day release) were implanted subcutaneously. Tumour growth was followed by visual inspection and bioluminescence measurements as described above.

Breast carcinoma model

Orthotopic tongue tumours were induced in the tip of the tongue through a submucosal injection of $4 \times 10^{4}$ OSC-19luc2-cGFP cells. When tumours were visible and bioluminescence signal ranged between $5 \times 10^{9}$ and $1 \times$ $10^{10}$ relative light units (RLU) imaging experiments started.

Peritonitis carcinomatosa

Multiple small MCF-luc2-cGFP tumours were induced in the peritoneum by intraperitoneal injection of $2.5 \times 10^{5}$ 
MCF-luc2-cGFP cells. Tumour growth was followed twice a week by bioluminescence as described above. Imaging experiments initiated when multiple tumour nodules were formed of various sizes.

\section{NIR fluorescence imaging systems}

Real-time NIR fluorescence imaging and operative resection of the tumours was performed using the next generation Artemis imaging system (Quest Medical Imaging, Middenmeer, the Netherlands). An earlier iteration of this system was extensively validated [30]. This system has a freely moveable handheld camera for simultaneous acquisition of visible light and NIR fluorescence. Since the system has been improved such that the camera can be fixed in a stable position with an arm, while both camera and arm are covered with a sterile drape. The illumination efficiency and homogeneity has been improved with a ring containing eight hemispheric illumination lenses centred around a wide field imaging lens for open surgery. Illumination is provided by four visible light sources with peaks centred in the blue, cyan, green and red and a NIR laser with a peak at $785 \mathrm{~nm}$ for fluorescence excitation. Reflected excitation light is blocked by a 750-800 nm notch filter. Captured visible and NIR light is split using a prism containing a dichroic coating $(<785 \mathrm{~mm})$. Visible light additionally passes through a low-pass filter $(<640 \mathrm{~nm})$ and the NIR emission light is filtered with a high pass filter $(>808 \mathrm{~nm})$. Exposure times and sensor gains are separately adjustable for both imaging channels. The visible light channel, the NIR fluorescence channel and an adjustable overlay of both channels are simultaneously presented during the procedures.

Next to the Artemis imaging system, the Pearl Impulse small animal imaging system (LI-COR) was used as a preclinical reference to visualize tumours and calculate the tumour-to-background ratios (TBRs). Data from the Artemis and Pearl imaging systems were analysed using imageJ (W. Rasband, Bethesda, Maryland) and the Pearl Cam Software, respectively.

\section{NIR fluorescence measurements}

Subcutaneous HT29 colon tumours were used to confirm in vivo EpCAM specificity of 323/A3-800CW and to measure fluorescence over time. When the subcutaneous HT29 colon tumours were $36 \pm 6 \mathrm{~mm}^{2}, 1 \mathrm{nmol}$ $(\approx 150 \mu \mathrm{g})$ of $323 / \mathrm{A} 3-800 \mathrm{CW}(n=3), 1 \mathrm{nmol}(\approx 150 \mu \mathrm{g})$ MOPC-800CW $(n=3)$ or $1 \mathrm{nmol}(\approx 1.1 \mu \mathrm{g})$ of $800 \mathrm{CW}$ carboxylate was injected intravenously. NIR fluorescence signals were measured at $0,4,24,48,72$ and $96 \mathrm{~h}$ after injection using the PEARL small animal imaging system and the intraoperative Artemis imaging system after which TBRs were calculated.

After in vivo confirmation of the EpCAM specificity and establishment of the optimal time frame for imaging with the subcutaneous model, the clinically more relevant orthotopic MCF-7-luc2-cGFP breast, OSC-19-luc2-cGFP tongue and HT29-luc2 colon tumours were evaluated. Hence, $1 \mathrm{nmol}$ of $323 / \mathrm{A} 3-800 \mathrm{CW}(n=3)$ or $1 \mathrm{nmol}$ MOPC21-800CW $(n=3)$ was intravenously injected in each group. Fluorescence imaging of mice bearing orthotopic tumours was performed $72 \mathrm{~h}$ after administration for optimal TBR as determined in the subcutaneous HT29 colon carcinoma model. TBRs of orthotopic tumours were measured and tumours were resected under NIR fluorescence guidance using the Artemis imaging system. Ex vivo, fluorescent measurements of resected tissue were performed on a back table. Tumours were sliced and fluorescence measurements were performed on the sections to evaluate the distribution of the probe. Resected tumours from the head-and-neck cancer and breast cancer models were assessed by BLI imaging and GFP fluorescence imaging (OSC-19-luc2-cGFP and MCF-7luc2-cGFP, IVIS spectrum).

The MCF-luc2-cGFP peritonitis carcinomatosa tumour model was used to determine the minimal tumour sizes that could be detected by intra-operative fluorescence imaging using the EpCAM specific antibody 323/A3$800 \mathrm{CW}$ in combination with 2 imaging systems. Because an enhanced permeability and retention (EPR) effect in these micrometases is not expected as indicated recently by Hall et al. [34] no MOPC21-800CW control was used in this model. Mice were anesthetized $72 \mathrm{~h}$ after intravenous injection of $323 / \mathrm{A} 3-800 \mathrm{CW}$ ( $1 \mathrm{nmol}, n=3)$, as described above and fluorescence imaging using the Pearl and Artemis imaging system was performed. A midline abdominal incision was made and the abdominal skin was removed. Fluorescence imaging of the mice with both imaging systems was performed followed by resection of the peritoneum and again fluorescence images were taken. Fluorescence imaging of the abdominal area was performed to search for residual intraperitoneal tumour nodules. Presence of tumour nodules and tumour specific NIR fluorescence was confirmed by bioluminescence imaging and GFP fluorescence imaging, as described before. Receiver Operator Curve (ROC) analysis was performed for the detection of micrometastases in the peritoneum. The overlay of the BLI and GFP signals was used as the ground truth for tumour metastases and the ascending TBRs as positive cut off criteria. The area under the curve (AUC), including the sensitivity and specificity rates at the optimal TBR cut off were computed. The signals of NIR fluorescence and the overlay with BLI and GFP was confirmed against pathology in the primary tumor but not in the micrometastases.

Multiple regions of interest were drawn in the tumour and in adjacent normal tissue and divided by each other to calculate TBRs. TBRs of subcutaneous colon and orthotopic breast tumours were calculated with skin overlying 
the tumour and adjacent normal tissue. Tongue tumours were imaged through an epithelial cell layer covering the tumour and normal tissue. For colon tumours the peritoneum was opened.

\section{In vivo competition study}

Three of the six nude Balb/C mice with bilateral orthotopic MCF-7-luc2-cGFP breast tumours were pre-injected with unconjugated 323/A3 antibody (1 mg, intraperitoneal, $100 \mu \mathrm{L}$ ). After $48 \mathrm{~h}$, all six mice were intravenously injected with $1 \mathrm{nmol} 323 / \mathrm{A} 3-800 \mathrm{CW}$. Then, $72 \mathrm{~h}$ after injection of 323/A3-800CW fluorescence imaging of all mice was performed using a Pearl imaging system. Mice were sacrificed and tumours were collected. Quantification of fluorescence was done as described before [37]. In brief, tumours were resected and lysed with a TissueLyser II system (Qiagen, Venlo, The Netherlands) using pre-cooled Eppendorf tube holders, 5-mm stainless steel beads, and RIPA buffer supplemented with a complete EDTA-free mini tablet protease inhibitor cocktail. Homogenates were serially diluted in 96-well plates, in parallel with a probe dilution. The fluorescence intensity of both series was detected at $800 \mathrm{~nm}$ using the Odyssey scanner. The concentration of probe in the homogenates was extrapolated from the calibration curves and the concentration values were used to calculate the injected dose per gram of tissue (\% ID/g) with standard error of the mean (SEM) indicated per group, based on the volume of the homogenate and the weight of the tumours.

\section{Histology and NIR fluorescence microscopy}

After ex vivo fluorescence measurements, tumours were snap frozen in isopentane and kept at $-80{ }^{\circ} \mathrm{C}$. Tissues were sectioned at $10 \mu \mathrm{m}$ and fluorescence imaging was performed using the Odyssey imager. The presence of OSC-19-luc2-cGFP and MCF-7-luc2-cGFP tumour cells was confirmed by fluorescence microscopy (Nikon Eclipse e800). All histologic sections were stained with standard haematoxylin-eosin stain (HE) after acetone fixation. To confirm the presence of HT29 and HT29-luc2 cells, sections were stained with an anti-human wide-spectrum cytokeratin antibody (Abcam inc., Cambridge, MA, USA). Primary antibodies or controls were incubated for $60 \mathrm{~min}$ at room temperature. All slides were three times washed with PBS and incubated with Envision anti-rabbit (DAKO) for $30 \mathrm{~min}$ at room temperature. Subsequently, the slides were washed with PBS and staining was visualized by using 3,3-diaminobenzidine. Sections were counterstained with haematoxylin, dehydrated and mounted with pertex. Frozen OSC-19-luc2-cGFP and MCF-7-luc2-cGFP tumours were stained with an anti-cGFP staining (Evrogen, Moscow, Russia). Sections stained with anti-cGFP were fixated with $4 \%$ formalin for $10 \mathrm{~min}$. After washing with PBS, cells were treated with $0,1 \%$ saponin/PBS for $10 \mathrm{~min}$ and incubated with the anti-GFP antibody, diluted in $0,1 \%$ saponin/PBS for $60 \mathrm{~min}$ at room temperature. Adjacent sections were fixated with aceton for 10 min followed by three washes with PBS and stained for cytokeratin as described before.

\section{Statistical analysis}

For statistical analysis, SPSS statistical software package (version 20.0 for Windows, IBM SPSS Inc, Chicago, USA) was used. TBRs were calculated by dividing the fluorescent signal of the tumour by fluorescent signal of surrounding healthy tissue. TBRs are reported in mean and standard deviation. A two-way repeated measurement ANOVA was used to assess the relation between TBRs in the dose groups and time points. Furthermore a paired Student's $t$-test was used to calculate the overall difference between the EpCAM specific and control groups. The two-way repeated measurement ANOVA was corrected using the Bonferroni correction. A $P$-value equal or lower than 0.05 was considered significant.

\section{Results \\ EpCAM expression on human cancer cell lines}

The colon adenocarcinoma cell line HT29 showed intermediate high expression of EpCAM while COLO320 hardly had any EpCAM expression $(200,000$ versus $<1000$ EpCAM/cell), see Fig. 1a). For MCF-7 breast cancer cells similar EpCAM over-expression was observed as for HT29, whereas considerably less expression was seen in MDA-MB-231 cells, as described previously (Fig. 1b). For head-and-neck cancer cells, the human hypopharyngeal squamous cell carcinoma cell line FaDu-luc2 and the oral squamous cell carcinoma cell line OSC-19 showed similar intermediate EpCAM expression (Fig. 1c). For all cancer cell lines the control antibody MOPC21 showed no significant signal. EpCAM expressing cell-lines MCF-7 and OSC19 were transfected with GFP and luciferase and were, next to HT29-luc2 used for further in vivo experiments.

\section{Conjugation, characterization and serum stability of 323/ A3-800CW}

Both the EpCAM specific (323/A3) and the control antibody (MOPC21) were labelled with IRDye 800CW (Fig. 2a) in comparable mean dye:antibody ratios of respectively $2.6 \pm 0.9$ and $2.8 \pm 0.9$, as determined spectrophotometrically, according to the protocol of the manufacturer of the dye. Plate assays containing both high and low EpCAM expressing cell lines were utilized to confirm EpCAM specificity of 323/A3-800CW after conjugation. A significant $(p<0.01)$ difference was observed between 323/A3-800CW and MOPC21-800CW signals on MCF-7, HT29, OSC-19 and FaDu cells, but not on the low EpCAM expressing COLO320 and MB-MDA-231 cells (Fig. 2b). The fluorescence intensity of MOPC21-800CW showed a slight concentration dependent increase as a result of non- 


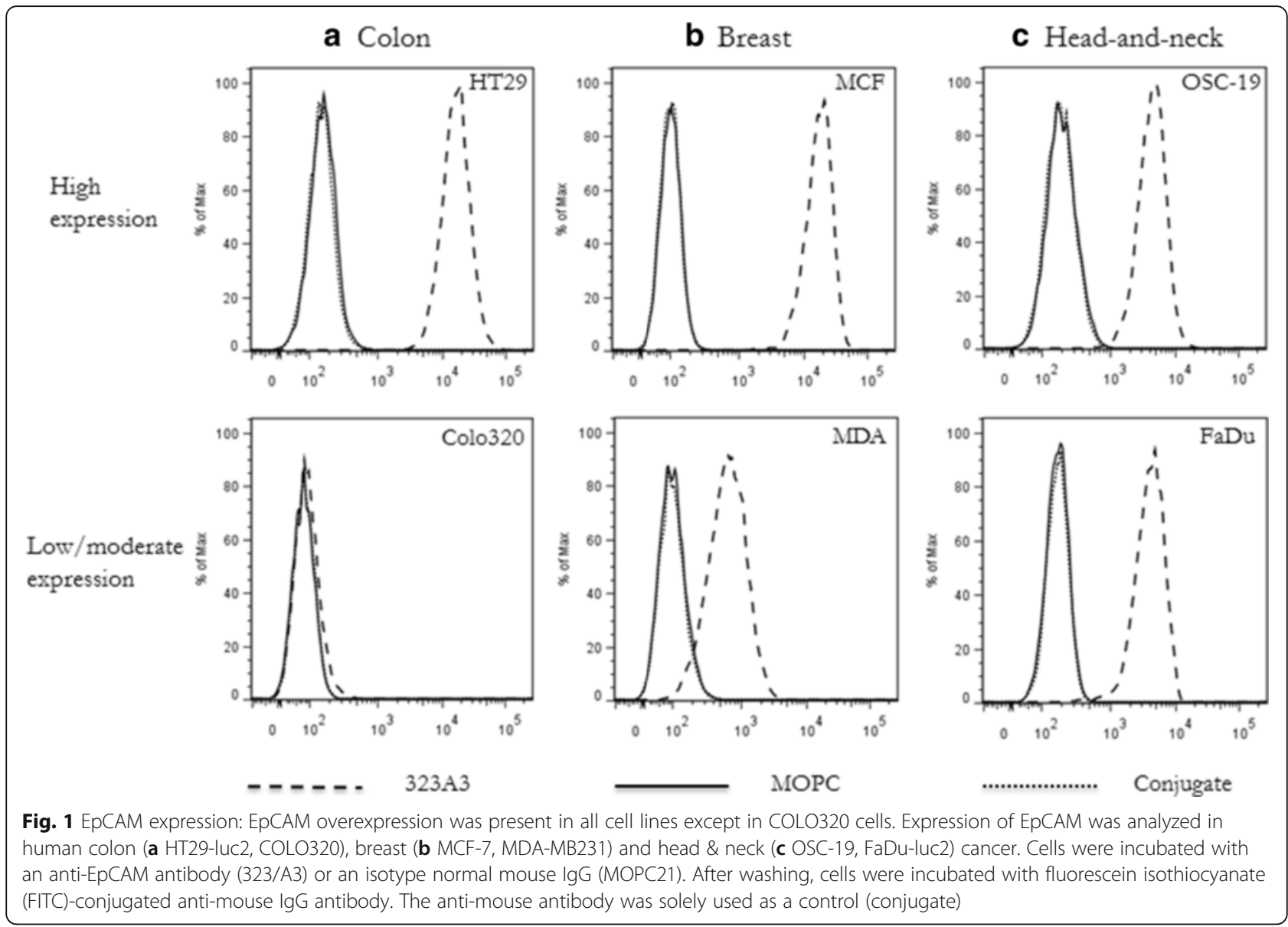

specific binding. The results are in accordance to the EpCAM expression found using flow cytometry results of the antibodies without $800 \mathrm{CW}$ label, as shown in Fig. 1. The increase in concentration resulted in a plateau in fluorescence intensity in MDA-MB-231, OSC-19 and $\mathrm{FaDu}$ cells. A further increase in concentration did not increase signal intensity. This phenomenon was not seen in MCF-7 and HT29 cells, possibly due to the high EpCAM expression. The EpCAM specific conjugate showed to be stable in human serum with more than $60 \%$ of the conjugate still free to bind EpCAM after $96 \mathrm{~h}$ with the remaining 40\% aggregated or bound to albumin (Fig. 2c).

\section{Intra-operative NIR fluorescence tumour delineation and resection}

In vivo specificity of 323/A3-800CW was shown by competition with unconjugated antibody. Pre-injection of $1 \mathrm{mg}$ unconjugated 323/A3, resulted in a decrease of the percentage of injected dose per gram of tumour from $3.2 \pm 0.9 \%$ $\mathrm{ID} / \mathrm{g}$ to $1.0 \pm 0.1 \% \mathrm{ID} / \mathrm{g}(p<0.01, n=3$ in both groups), see Fig. 3a. The HT29 subcutaneous tumour model was used to assess the feasibility of intraoperative fluorescence delineation using $1 \mathrm{nmol}$ (150ug) intravenous 323/A3$800 \mathrm{CW}$ combined with the Artemis imaging system.
Tumours could be clearly delineated from $4 \mathrm{~h}$ post injection and an increase in TBR was seen with highest values at $72 \mathrm{~h}$ post-injection $(5.2 \pm 0.7$, Fig. $3 \mathrm{~b})$. After $72 \mathrm{~h}$ the TBR slightly decreased. Significant differences in TBR $(p<0.01)$ between EpCAM specific (323/A3$800 \mathrm{CW})$ and non-specific control (MOPC21-800CW) could be observed from $24 \mathrm{~h}$ post injection (TBR $3.2 \pm$ 0.1 and $1.7 \pm 0.2$ respectively). MOPC21-800CW and IRDye $800 \mathrm{CW}$ showed a significantly lower TBR at all time points $(p<0.01)$.

Based on the results from the subcutaneous tumour model an optimal incubation time of $72 \mathrm{~h}$ was used to evaluate NIR fluorescence in clinically more realistic orthotopic mouse models for colon, breast and head-andneck cancer. After exploration of the tumour, we assessed the feasibility of fluorescence-guided resection and chose suitable resection margins with the real-time NIR fluorescence feedback of 323/A3-800CW and the Artemis imaging system. All tumours were radically resected as confirmed by BLI evaluation. Significant differences $(p<0.01)$ in TBRs were observed between the orthotopic colon (HT29) mice injected with 323/A3-800CW (TBR 13.5 \pm 3.6 ) and MOPC21-800CW (TBR $1.8 \pm 0.4$ ) (Fig. 3c). For the breast (MCF-7) and head-and-neck (OSC-19) cancer 

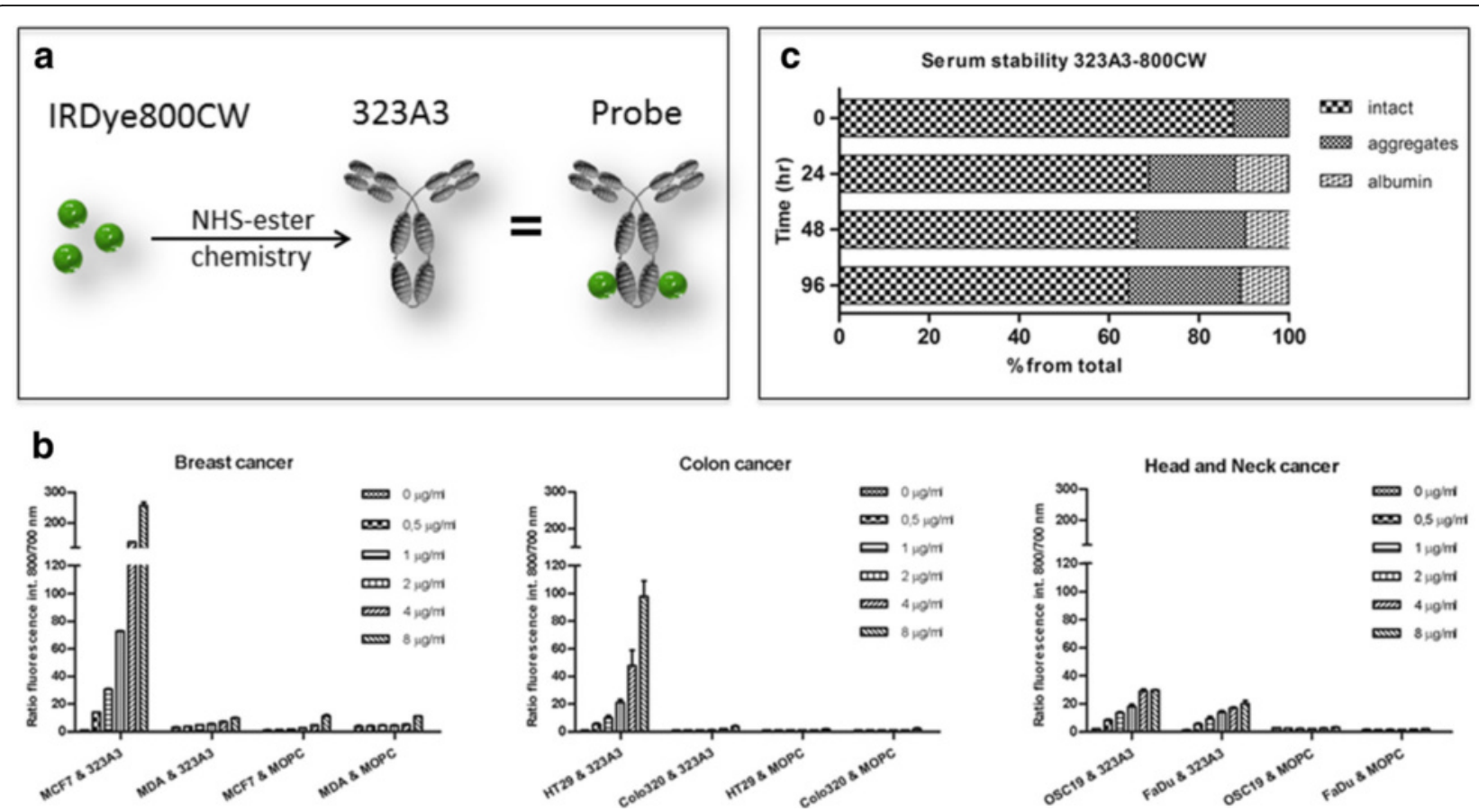

Fig. 2 Conjugation, serum stability and EpCAM specificity: Conjugation of IRDye 800CW to 323/A3 and MOPC21 was done through NHS ester conjugation. A mean labeling ratio of $2.6 \pm 0.98$ and $2.8 \pm 0.99$ was obtained for 323/A3 and MOPC21 respectively a. EpCAM specificity of 323/A3 was confirmed after conjugation of IRDye $800 \mathrm{CW}$ on various cell types, corrected for cell number using the $800 / 700 \mathrm{~nm}$ ratio $\mathbf{b}$. Experiments were done in human colon (HT29-luc2, COLO320), breast (MCF-7-luc2-cGFP, MDA-MB231) and head \& neck (OSC-19-luc2-cGFP, FaDu-luc2) cancer. Cells were incubated with different concentrations of 323/A3-800CW or an isotype normal mouse lgG (MOPC21) conjugated to 800CW. To correct for the number of cells, the ratio of NIR fluorescence and the fluorescence intensity of TO-PRO-3 was plotted. 323/A3-800CW showed to be stable in human serum with more than $60 \%$ of the conjugate still free to bind EpCAM after $96 \mathrm{~h}$ with the remaining $40 \%$ aggregated or bound to albumin c

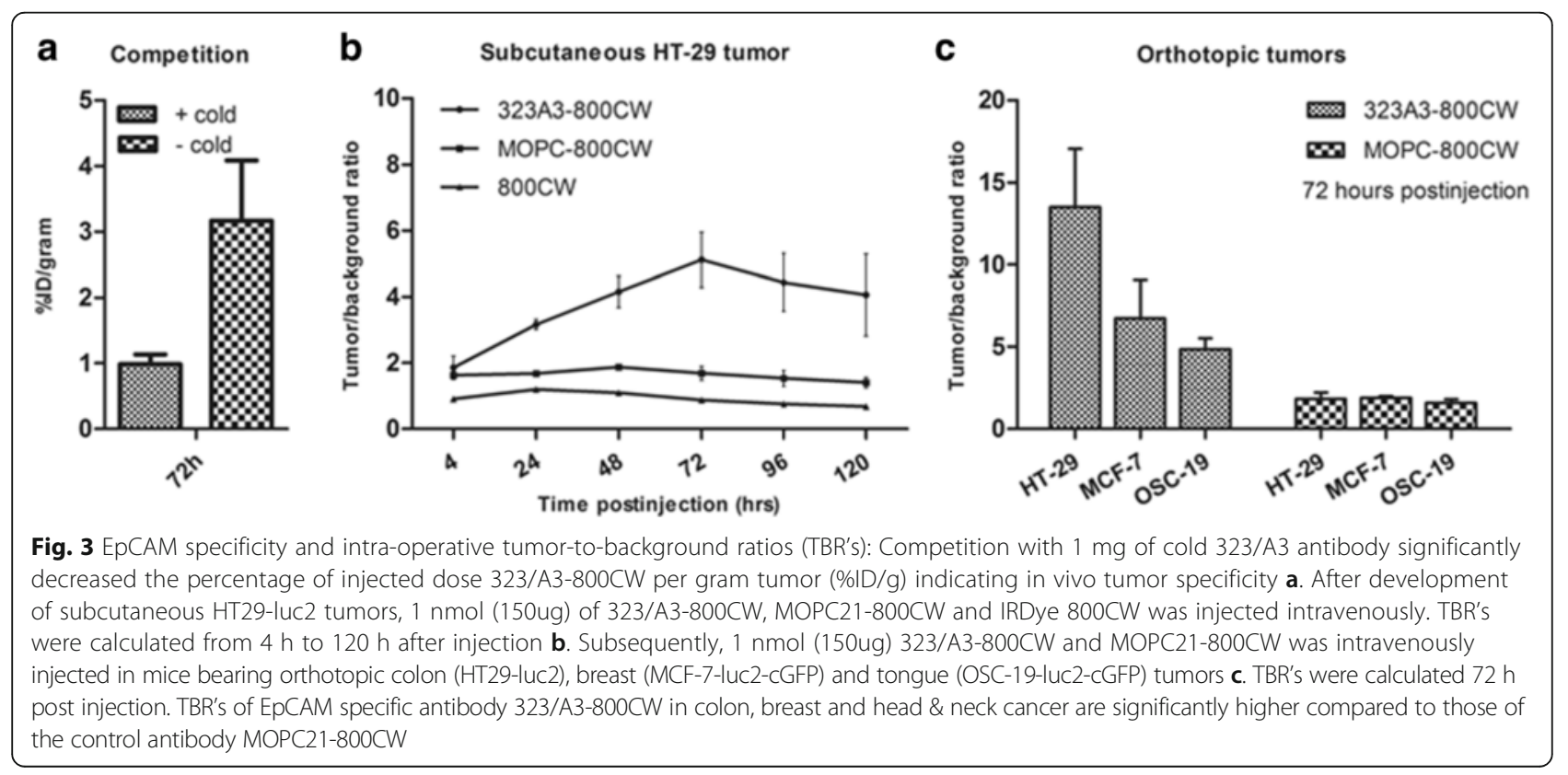


models, 323/A3-800CW showed TBRs of $6.7 \pm 1.9$ and $4.9 \pm 0.7$ respectively (Fig. 3c). Significantly lower TBR values of $1.9 \pm 0.1$ and $1.6 \pm 0.2$ were observed for MOPC21-800CW in breast and head-and-neck cancer respectively $(p<0.01)$. Examples of in vivo and ex vivo images that were acquired with the Artemis imaging system after injection of 323/A3-800CW or MOPC21$800 \mathrm{CW}$ are shown for the orthotopic colon (Fig. 4a and b), breast (Fig. 4c) and head-and-neck cancer model (Fig. 4d). Camera exposure time and gain were intra-operatively adjusted to obtain optimal image contrast. The most important factors influencing measured fluorescent intensities were sample fluorescence intensity, camera settings and the coupled distance to sample of both illumination source and camera. The Artemis camera is not calibrated for absolute intensity measurements (e.g. in lumen), hampering absolute intensity comparisons between images.

\section{Detection of small tumour sizes}

Four weeks after the intra-peritoneal injection of MCF-7luc2-cGFP cells multiple tumours were observed by BLI imaging. Millimetre size tumour nodules located on the peritoneum, which were not visual by the naked eye, could be observed by NIR fluorescence imaging after injection of $1 \mathrm{nmol}$ (150ug) 323/A3-800CW, using both the Pearl and Artemis imaging system (Fig. 5). After the peritoneum was resected, BLI confirmed that intra-peritoneal metastases could be detected by NIR fluorescence imaging (Fig. 5a). As in Fig. 4, camera exposure time and gain were adjusted to obtain optimal contrast. ROC curve analysis showed an accuracy of $98 \%$ (area under the curve, $p<0.0001$ ) for detecting micro metastases with a sensitivity of $93 \%$ and specificity of $92 \%$ at the curve's optimal TBR cut off value (Fig. 5). A cut off value obtaining the highest sensitivity of $100 \%$ correlates with a specificity of $73 \%$. a
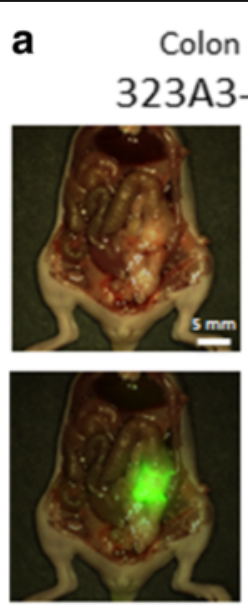

C
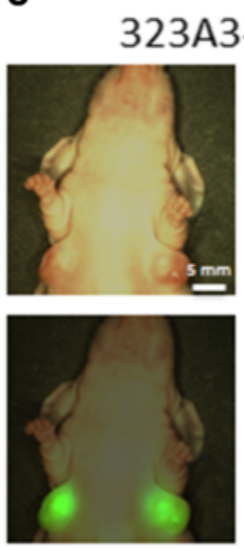

Breast cancer
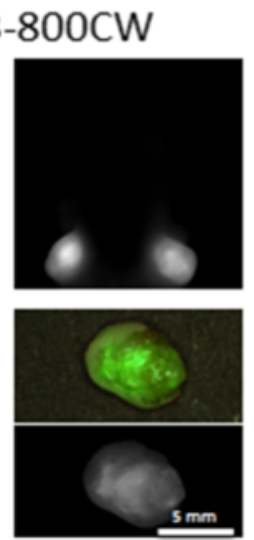

b

Colon cancer MOPC-800CW
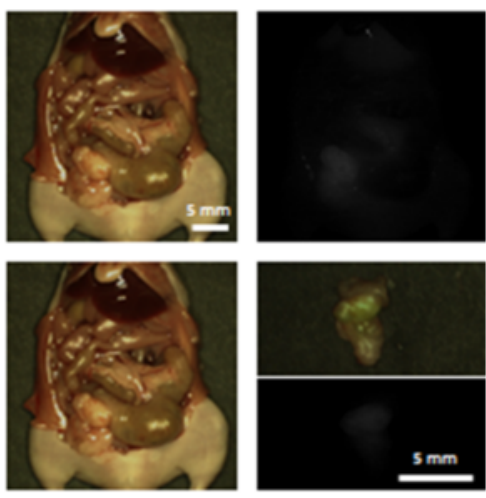

d Head \& Neck cancer 323A3-800CW
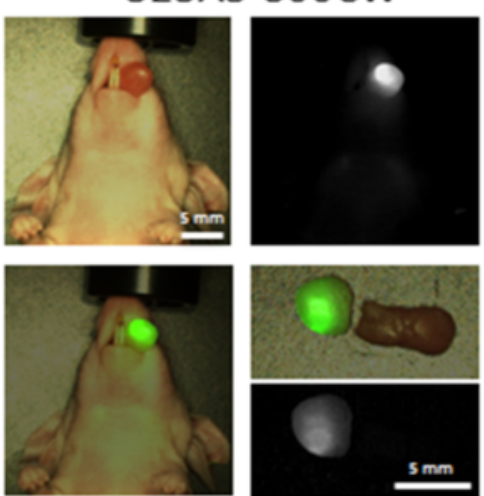

Fig. 4 Intra-operative NIR fluorescence delineation of colon, breast and Head \& Neck cancer: Colon a, b breast c and head \& neck tumors $\mathbf{d}$ could clearly be visualized during operation using EpCAM specific 323/A3-800CW (TBR colon 13.5, breast 6.7 and Head-and-neck 4.9) and the Artemis imaging system. 323/A3-800CW (A, C, D, 1 nmol) and the non-specific antibody MOPC21-800CW (B, 1 nmol) were intravenously injected. After an incubation of $72 \mathrm{~h}$ NIR fluorescence imaging was performed. Depicted images are: bright light, fluorescence, overlay (fluorescence and bright light), ex vivo fluorescence and ex vivo overlay. Scale bars are $5 \mathrm{~mm}$. Artemis fluorescence intensities were intra-operatively optimized for optimal contrast. Intensities can only be compared within images 


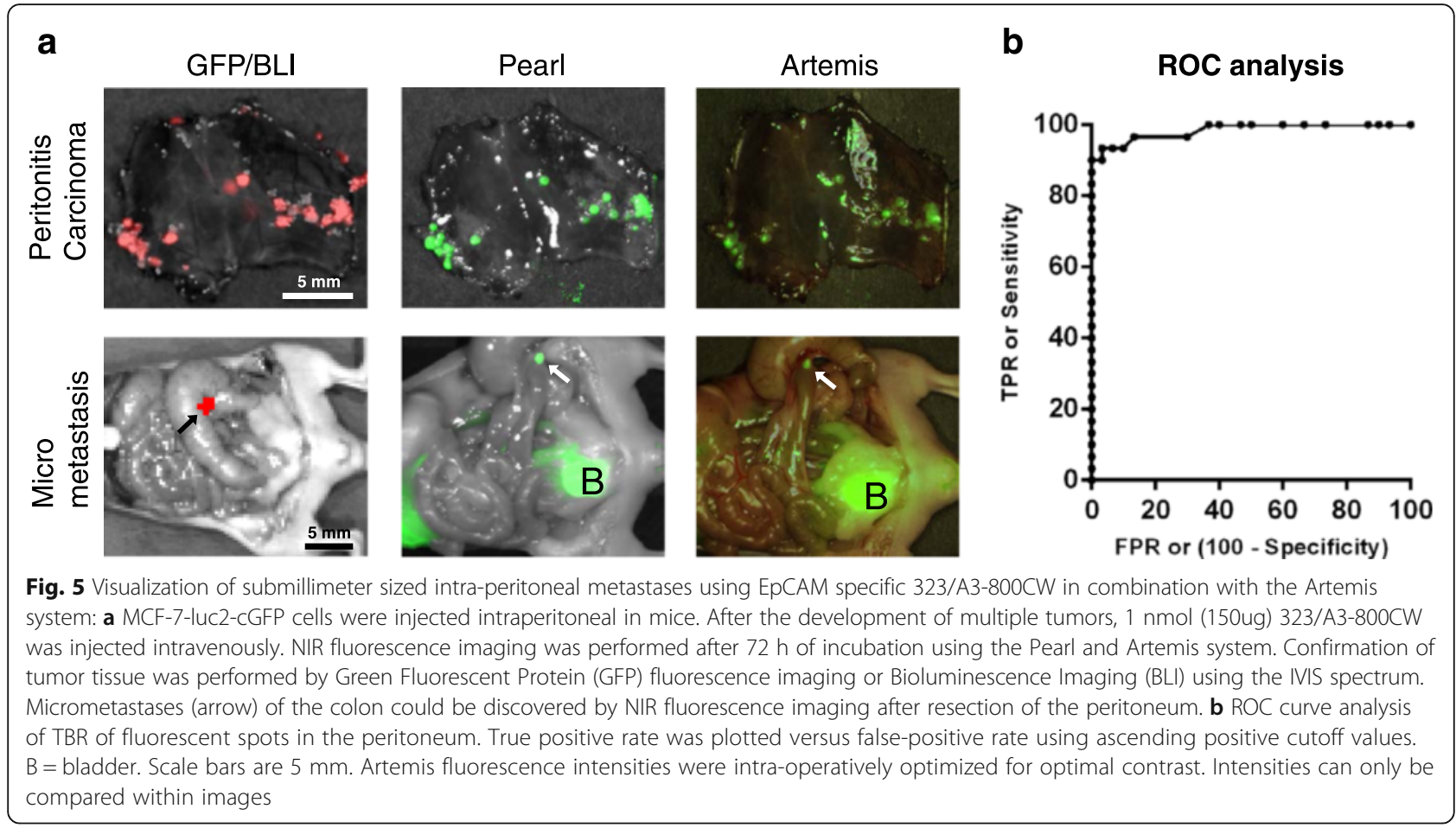

A specificity of $100 \%$ on the other hand correlates with a sensitivity of $84 \%$.

\section{Histology and Immunohistochemistry}

Orthotopic tumour tissue was harvested $72 \mathrm{~h}$ after injection of 323/A3-800CW, MOPC21-800CW and IRDye 800CW. In sections of cGFP transfected breast and tongue tumours, the presence of tumour tissue was confirmed by anti-cGFP immunohistochemical staining (brown colour, Fig. $6 \mathrm{c}$ and d). Fig. 6a clearly illustrates NIR fluorescence (indicated in red) intensity and localization of $323 / \mathrm{A} 3-800 \mathrm{CW}$, MOPC-800CW and IRDye $800 \mathrm{CW}$ in tissue sections of tongue tumours, surrounded by normal tissue of the tongue. Fluorescence of 323/A3-800CW co-localized with the outer part of the tumour bulk and with small peninsulas of tumour cells (Fig. 6a). Fluorescence intensity of MOPC21-800CW and IRDye $800 \mathrm{CW}$ was hardly visible and did not show association with tumour tissue (Fig. 6b). In both, breast and colon cancer tissue sections NIR fluorescence appeared in the outer rim of tumour tissue. (Fig. 6c and d). In colon cancer clear colocalization of small tumour islands and NIR fluorescence (indicated in red) was observed with 323/A3$800 \mathrm{CW}$ (Fig. 6d). Cytokeratin staining (brown) was performed to confirm the presence of colonic tumour cells in sections of orthotopic colon tumours (Fig. 6d).

\section{Discussion}

In this study we investigated an EpCAM-specific NIRfluorescent agent using a state-of-the-art next generation clinical NIR imaging system. The recent technical developments stimulate the use of advanced but affordable imaging systems in the clinic. The Artemis imaging system simultaneously acquires NIR-fluorescent and visible light, generating a merged image in real-time. Systems like this will boost the demand for specific tumour probes dramatically.

The EpCAM directed antibody in this study has been extensively evaluated for imaging of various tumour models using radionuclides Technetium, Zirconium, Iodium and Rhenium [28, 29, 38]. Typically, in these experiments 15-20 $\mu \mathrm{g}$ of conjugate was used per mouse to obtain clear tumour signals in subcutaneous breast, colon and ovarian tumours. Using the same antibody but conjugated to a NIR dye and in combination with a state of the art NIR-fluorescence imager, we showed that $1 \mathrm{nmol}$ $(=150 \mu \mathrm{g})$ clearly identified breast, colon and head-andneck cancers, as well as micro-metastases in human orthotopic xenograft mouse models. The probe/system combination allowed an accurate demarcation of the tumours, the recognition of tumour margins, examination of malignant spread, and identification of micrometre sized metastasis or remnant disease.

A probe against a 'universal' target facilitates clinical usability, and prevents time consuming and costly development of multiple agents. Although EpCAM is considered to be a potential target for epithelial derived cancers, not all epithelial cancers over-express EpCAM enough to outflank adjacent normal tissue. But on the other hand not all tumours of mesenchymal origin are EpCAM negative. Over-expression of EpCAM was recently observed in all 


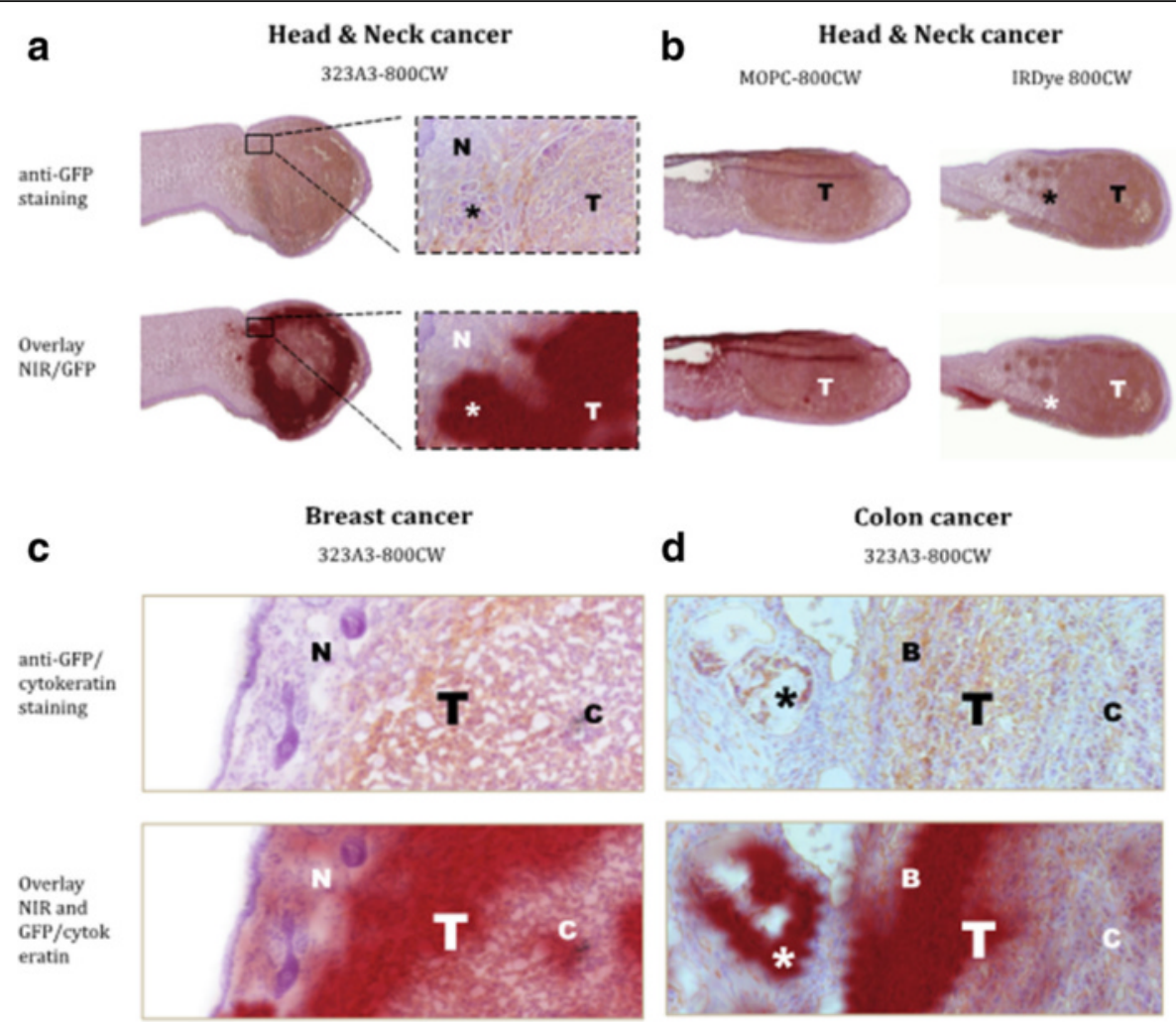

Fig. 6 Histology: 323/A3-800CW is specifically located in head-and-neck $\mathbf{a}$, b, breast c, and colon $\mathbf{d}$ tumors. Tumor tissue was sectioned 72 h after injection of 323/A3-800CW, MOPC21-800CW or IRDye 800CW. Shown are anti-GFP (brown, head-and-neck cancer, breast cancer) or cytokeratin (brown, colon cancer) immunohistochemistry stainings and overlays of NIR fluorescence (red) and anti-GFP/cytokeratin stainings. In all tissue a clear overlap is seen between NIR fluorescence from 323/A3-800CW and anti-GFP immunohistochemistry staining (indicating tumor). NIR fluorescence is mainly located in the border of tumors and even small tumor islands $\left(^{*}\right)$ are NIR fluorescent. A low, non specific fluorescence signal was observed in sections with MOPC21-800CW and IRDye 800CW (B). T = tumor; $\mathrm{N}=$ normal tissue; $\mathrm{C}=$ center tumor; $\mathrm{B}=$ tumor border. $\mathrm{A}$ and $\mathrm{B}$ $1 \times$ zoom; C and D 10× zoom

osteosarcomas, half of the angiosarcomas and $62.5 \%$ of the leiomyosarcomas, indicating the broad spectrum of tumours that might be targeted [39].

In breast cancer, $20 \%$ of tumours that seem radically resected during surgery turn out to be irradically removed in histologic analyses. Consequently, FGS in breast cancer could enhance the number of radical resections without increasing margins, thus improving patient's prognoses and cosmetic outcomes after lumpectomies and oncoplastic surgery $[3,40]$. As pointed out earlier, in copies per cell EpCAM can probably not compete with Her2/Neu in the subpopulation of Her2 positive breast cancers, but in percentage of positive breast tumours EpCAM is clearly the more prevalent target. For head-and-neck cancer a relatively low percentage of tumours is irradically resected (16\%). Due to the many vital structures in the surrounding tissue, resection margins should be small and FGS targeting would be of great clinical benefit. EpCAM overexpression is seen in $62.5 \%$ of tongue tumours $[23,41]$. In colorectal cancers $80-100 \%$ overexpression of EpCAM is found.
Although irradical resections occur, the main problem is damage to vital structures in the lower abdomen. Ureteral injury is a rare but serious complication of lower abdominal surgery, with a reported incidence varying from 0.7 up to $10 \%$ [42]. EpCAM-based FGS of colorectal tumours in combination with FGS of the ureters could enhance the number of radical resections while preserving vital structures.

In our study, we performed a single injection of $1 \mathrm{nmol}$ (150ug) of $323 / \mathrm{A} 3-800 \mathrm{CW}$ to visualize tumours. This is the equivalent of $0.5 \mathrm{mg} / \mathrm{kg}$ for humans when converted using the body-surface-area method [43]. Using this dose, subcutaneously located colon tumours were clearly recognized from $4 \mathrm{~h}$ after injection with optimal TBRs at $72 \mathrm{~h}$, showing the feasibility of the agent. The 72-h timepoint was utilized to perform surgery at the orthotopic colon, breast, and head-and-neck cancer models validating the results from the subcutaneous model in clinically more relevant models. At this time point the agent-imaging system combination was able to indicate small tumour 
nodules on the peritoneum or in the abdominal cavity that were otherwise only detectable by bioluminescence of these cells. Although we did not study the lower boundary of tumour size that could be detected, tumour nodules of $1 \mathrm{~mm}^{3}$ could clearly be visualized. Based on the number of cells in spheroids of that size, we estimate the minimal number of cells to be detectable above 50,000. Obviously this detection limit is depending on multiple factors like the imaging system, optical properties of overlying tissue and the probe. Although ROC analysis for the detection of micrometastases showed an excellent sensitivity and specificity at the optimal TBR cut off, it should be noted that the ex vivo detection of micrometastases in the peritoneum is very opportune, because almost no background fluorescence is observed in the peritoneum and the detection of metastases is not compromised by any tissue depth. Due to the lack of performance standards for clinically applicable imaging systems as well as imaging agents, at present, the combination of both is of major importance for a successful outcome $[44,45]$.

Although the anti-EpCAM/800CW conjugate performed well in various tumour types, this probe could still be optimized. The use of antibodies with relatively high affinity, like 323/A3, might culminate in heterogeneous tumour staining, concentrated around the tumour vascularization rather than homogeneous throughout the tumour. The use of an antibody with low or intermediate EpCAM affinity for EpCAM might promote a more homogenous distribution and avoid adverse effects at the cost of a lower total tumour uptake $[29,46]$. An improvement in imaging accuracy without compromising the total tumour uptake could be expected by reducing $\mathrm{Fc} / \mathrm{Fc} \gamma \mathrm{R}$ interactions through deglycosylation of antibody-based imaging as recently demonstrated by Gao et al. [47]. Further, most EpCAM antibodies target actually the same EGF-like domain of EpCAM. Antibodies identifying other EpCAM domains, like the $\mathrm{C}$ - domains might improve detection efficiency [48]. The use of any mouse-derived antibody, like 323/A3, will inevitably induce human anti-mouse antibodies. Although this is not disastrous for imaging purposes, where only one single dose is needed, a chimerized or humanized version with retained efficacy should be preferred [29]. Last but not least, fragments of antibodies like Fab or Fab2 could have higher penetration capabilities compared to full-size antibodies, as shown for several antibodies including anti-EpCAM [35, 38, 49]. Using antibody fragments would lead to shorter incubation times, making clinical translation more applicable. Moreover, recent developments have opened the way for hybrid molecules like immunoenzymosomes, consisting of liposomes equipped with 323/A3 Fab fragments for targeting [50]. Alternative probes for pre- and intraoperative imaging could also be generated by conjugation of the antibody with 2 different labels, one for PET/SPECT and one for NIRF imaging like has been done recently $[34,35,51,52]$.

\section{Conclusions}

FGS is a promising technique to ensure intra-operative fluorescence feedback of tumour margins. Clinical success is only achieved by using a dedicated camera system and tumour-specific agents. This study aimed to achieve complete preclinical validation of an EpCAM targeting fluorescence agent in combination with a next-generation Artemis imaging system. We showed the ability to visualize, primary tumours and millimetre sized tumour nodules and metastases that were otherwise invisible for the human eye. As a novel EpCAM specific optical agent can be used in a wide variety of tumours, together with the knowledge from previous clinical trials and the results from this study, this paves the way for a fast and cost effective clinical translation.

\section{Abbreviations \\ BLI: Bioluminescence imaging; EGFR: Epidermal growth factor receptor: EpCAM: Epithelial cell adhesion molecule; FGS: Fluorescence-guided surgery; NIR: Near-infrared; PBS: Phosphate buffered saline; ROC: Receiver operator characteristic; TBR: Tumor-to-background ratio; VEGF(R): Vascular endothelial growth factor (receptor)}

\section{Funding}

CTMM (grant MUSIS 03O-202), CTMM (grant DECODE 03O-202-04), Dutch Cancer Society (grants UL2010-4732 and UL2015-7642), H2020-MSCA-RISE (grant PRISAR 644373)

\section{Availability of data and materials \\ The data will not be shared.}

\section{Authors' contributions}

$P \vee D, M B, S K, B L, J D, C v d V, P K, A V, C L, C S$ designed the study. PvD, MB, HP, MvdG, TS, QT, RC, AF, performed experiments and/or gave technical support. $P \vee D, M B$, analysed data and prepared figures. PvD, MB, CS wrote the manuscript. All authors read and approved the final manuscript.

\section{Competing interests}

All authors declare that they have no competing interests.

\section{Consent for publication}

Not applicable.

\section{Ethics approval and consent to participate}

The local Animal Welfare Committee of the Leiden University Medical Centre approved the study design and all animal experiments.

\section{Author details}

'Department of Radiology, Division of Molecular Imaging, Leiden University Medical Centre, Leiden, Netherlands. Percuros BV, Enschede, The Netherlands. ${ }^{3}$ Department of Radiology and Division of Image Processing, Leiden University Medical Centre, Leiden, Netherlands. ${ }^{4}$ Department of Surgery, Leiden University Medical Centre, Leiden, Netherlands. ${ }^{5}$ Department of Otorhinolaryngology and Head and Neck Surgery, Erasmus Medical Centre, Rotterdam, Netherlands. ${ }^{6}$ Department of Immunohematology and Blood Transfusion, Leiden University Medical Centre, Leiden, Netherlands. ${ }^{7}$ Division of Biochemistry, Netherlands Cancer Institute, Amsterdam, Netherlands. ${ }^{8}$ Department of Pathology, Leiden University Medical Centre, Leiden, Netherlands. ${ }^{9}$ Antibodies for Research Applications BV, Gouda, The Netherlands.

Received: 28 February 2016 Accepted: 30 October 2016 Published online: 14 November 2016 


\section{References}

1. Weissleder R, Pittet MJ. Imaging in the era of molecular oncology. Nature. 2008;452(7187):580-9.

2. Quirke $P$, Durdey $P$, Dixon MF, Williams NS. Local recurrence of rectal adenocarcinoma due to inadequate surgical resection. Histopathological study of lateral tumour spread and surgical excision. Lancet. 1986; 2(8514):996-9.

3. Rizzo M, lyengar R, Gabram SG, et al. The effects of additional tumor cavity sampling at the time of breast-conserving surgery on final margin status, volume of resection, and pathologist workload. Ann Surg Oncol. 2010;17(1):228-34.

4. Wong LS, Mcmahon J, Devine J, et al. Influence of close resection margins on local recurrence and disease-specific survival in oral and oropharyngeal carcinoma. Br J Oral Maxillofac Surg. 2012;50(2):102-8.

5. Xiong L, Gazyakan E, Yang W, et al. Indocyanine green fluorescence-guided sentinel node biopsy: a meta-analysis on detection rate and diagnostic performance. Eur J Surg Oncol. 2014;40(7):843-9.

6. Verbeek FP, Schaafsma BE, Tummers QR, et al. Optimization of near-infrared fluorescence cholangiography for open and laparoscopic surgery. Surg Endosc. 2014:28(4):1076-82.

7. Vahrmeijer AL, Hutteman M, Van der Vorst JR, Van de Velde CJ, Frangioni JV. Image-guided cancer surgery using near-infrared fluorescence. Nat Rev Clin Oncol. 2013;10(9):507-18

8. Sevick-Muraca EM, Sharma R, Rasmussen JC, et al. Imaging of lymph flow in breast cancer patients after microdose administration of a near-infrared fluorophore: feasibility study. Radiology. 2008;246(3):734-41.

9. Unno N, Nishiyama M, Suzuki M, et al. Quantitative lymph imaging for assessment of lymph function using indocyanine green fluorescence lymphography. Eur J Vasc Endovasc Surg. 2008;36(2):230-6.

10. Keereweer $S$, Van Driel PB, Snoeks TJ, et al. Optical image-guided cancer surgery: challenges and limitations. Clin Cancer Res. 2013;19(14):3745-54

11. Gioux S, Choi HS, Frangioni JV. Image-guided surgery using invisible near-infrared light: fundamentals of clinical translation. Mol Imaging. 2010;9(5):237-55.

12. Gong H, Kovar JL, Cheung L, Rosenthal EL, Olive DM. A comparative study of affibody, panitumumab, and EGF for near-infrared fluorescence imaging of. Cancer Biol Ther. 2013;15(2):185-93.

13. Persson M, Kjaer A. Urokinase-type plasminogen activator receptor (UPAR) as a promising new imaging target: potential clinical applications. Clin Physiol Funct Imaging. 2013;33(5):329337.

14. Zielinski R, Hassan M, Lyakhov I, et al. Affibody-DyLight conjugates for in vivo assessment of HER2 expression by near-infrared optical imaging. PLoS One. 2012;7(7):e41016

15. Paudyal B, Paudyal P, Shah D, Tominaga H, Tsushima Y, Endo K. Detection of vascular endothelial growth factor in colon cancer xenografts using bevacizumab based near infrared fluorophore conjugate. J Biomed Sci. 2014;21(1):35.

16. Lee $\mathrm{H}$, Kim J, Kim H, Kim Y, Choi Y. A folate receptor-specific activatable probe for near-infrared fluorescence imaging of ovarian cancer. Chem Commun (Camb). 2014;50(56):7507-10.

17. Jin $Z \mathrm{H}$, Josserand $\mathrm{V}$, Foillard $\mathrm{S}$, et al. In vivo optical imaging of integrin alphaV-beta3 in mice using multivalent or monovalent CRGD targeting vectors. Mol Cancer. 2007;6:41.

18. Winter MJ, Nagtegaal ID, van Krieken JH, Litvinov SV. The epithelial cel adhesion molecule (Ep-CAM) as a morphoregulatory molecule is a tool in surgical pathology. Am J Pathol. 2003;163(6):2139-48.

19. Went PT, Lugli A, Meier $S$, et al. Frequent EpCam protein expression in human carcinomas. Hum Pathol. 2004;35(1):122-8.

20. Spizzo G, Obrist P, Ensinger $C$, et al. Prognostic significance of Ep-CAM AND Her-2/neu overexpression in invasive breast cancer. Int J Cancer. 2002;98(6):883-8.

21. Osta WA, Chen Y, Mikhitarian $\mathrm{K}$, et al. EpCAM is overexpressed in breast cancer and is a potential target for breast cancer gene therapy. Cancer Res. 2004:64(16):5818-24.

22. Gastl G, Spizzo G, Obrist P, Dunser M, Mikuz G. Ep-CAM overexpression in breast cancer as a predictor of survival. Lancet. 2000;356(9246):1981-2.

23. Laimer K, Fong D, Gastl G, et al. EpCAM expression in squamous cell carcinoma of the oral cavity: frequency and relationship to clinicopathologic features. Oral Oncol. 2008:44(1):72-7.

24. Fong D, Moser P, Krammel C, et al. High expression of TROP2 correlates with poor prognosis in pancreatic cancer. Br J Cancer. 2008;99(8):1290-5.

25. Goossens-Beumer IJ, Zeestraten EC, Benard A, et al. Clinical prognostic value of combined analysis of Aldh1, Survivin, and EpCAM expression in colorectal cancer. Br J Cancer. 2014;110(12):2935-44.
26. Munz M, Murr A, Kvesic M, et al. Side-by-side analysis of five clinically tested anti-EpCAM monoclonal antibodies. Cancer Cell Int. 2010;10(1):44.

27. Gires O, Bauerle PA. EpCAM as a target in cancer therapy. J Clin Oncol. 2010;28(15):e239-40.

28. Meijs WE, Haisma HJ, Klok RP, et al. Zirconium-labeled monoclonal antibodies and their distribution in tumor-bearing nude mice. J Nucl Med. 1997:38(1):112-8.

29. Kievit E, van Gog FB, Schluper HM, van Dongen GA, Pinedo HM, Boven E. [186Re]-labeled mouse and chimeric monoclonal antibody 323/A3: a comparison of the efficacy in experimental human ovarian cancer. Nucl Med Biol. 1998;25(1): $37-45$.

30. Van Driel PB, Van de Giessen M, Boonstra MC, et al. Characterization and Evaluation of the Artemis Camera for Fluorescence-Guided Cancer Surgery. Mol Imaging Biol. 2015;6(16):413-23.

31. Van Driel PB, Van der Vorst JR, Verbeek FP, et al. Intraoperative fluorescence delineation of head and neck cancer with a fluorescent anti-epidermal growth factor receptor nanobody. Int J Cancer. 2014;134(11):2663-73.

32. Edwards DP, Grzyb KT, Dressler LG, et al. Monoclonal antibody identification and characterization of a Mr 43,000 membrane glycoprotein associated with human breast cancer. Cancer Res. 1986;46(3):1306-17.

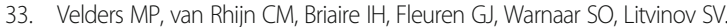
Immunotherapy with low and high affinity monoclonal antibodies 17-1A and 323/A3 in a nude mouse xenograft carcinoma model. Cancer Res. 1995;55(19): 4398-403.

34. Hall MA, Pinkston KL, Wilganowski N, et al. Comparison of mAbs Targeting Epithelial Cell Adhesion Molecule for the Detection of Prostate Cancer Lymph Node Metastases with Multimodal Contrast Agents: Quantitative Small-Animal PET/CT and NIRF. J Nucl Med. 2012:53(9):1427-37.

35. Eder M, Knackmuss S, Le GF, et al. 68Ga-labelled recombinant antibody variants for immuno-PET imaging of solid tumours. Eur J Nucl Med Mol Imaging. 2010;37(7):1397-407.

36. Tseng W, Leong X, Engleman E. Orthotopic mouse model of colorectal cancer. J Vis Exp. 2007;10:484

37. Oliveira S, Cohen R, Walsum MS, Van Dongen GA, Elias SG, Van Diest PJ, Mali W. Van Bergen en Henegouwen PM: A novel method to quantify IRDye800CW fluorescent antibody probes ex vivo in tissue distribution studies. EJNMMI Res. 2012;2(1):50.

38. Pak KY, Nedelman MA, Fogler WE, et al. Evaluation of the 323/A3 monoclonal antibody and the use of technetium-99 m-labeled 323/A3 Fab' for the detection of pan adenocarcinoma. Int J Rad Appl Instrum B. 1991;18(5):483-97.

39. Ward K, Amaya C, Verma K, et al. Epithelial cell adhesion molecule is expressed in a subset of sarcomas and correlates to the degree of cytological atypia in leiomyosarcomas. Mol Clin Oncol. 2015;3(1):31-6.

40. McLaughlin SA. Surgical management of the breast: breast conservation therapy and mastectomy. Surg Clin North Am. 2013;93(2):411-28.

41. Yanamoto S, Kawasaki G, Yoshitomi I, Iwamoto T, Hirata K, Mizuno A. Clinicopathologic significance of EpCAM expression in squamous cell carcinoma of the tongue and its possibility as a potential target for tongue cancer gene therapy. Oral Oncol. 2007;43(9):869-77.

42. Verbeek FP, Van der Vorst JR, Tummers QR, et al. Near-Infrared Fluorescence Imaging of Both Colorectal Cancer and Ureters Using a Low-Dose Integrin Targeted Probe. Ann Surg Oncol. 2014:21 Suppl 4:528-37.

43. Reagan-Shaw $S$, Nihal M, Ahmad N. Dose translation from animal to human studies revisited. FASEB J. 2008;22(3):659-61.

44. Zhu B, Sevick-Muraca E. A review of performance of near-infrared fluorescence imaging devices used in clinical studies. Br J Radiol. 2014;20:20140547.

45. Sevick-Muraca EM, Zhu B. The need for performance standards in clinical translation and adoption of fluorescence molecular imaging. Med Phys. 2013:40(4):040402

46. Thurber GM, Schmidt MM, Wittrup KD. Antibody tumor penetration: transport opposed by systemic and antigen-mediated clearance. Adv Drug Deliv Rev. 2008:60(12):1421-34

47. Gao P, Pinkston KL, Wilganowski N, et al. Deglycosylation of $\mathrm{mAb}$ by EndoS for Improved Molecular Imaging. Mol Imaging Biol. 2014;17(2):195-203.

48. Takao M, Nagai Y, Torii T. Cysteine-poor region-specific EpCAM monoclonal antibody recognizing native tumor cells with high sensitivity. Monoclon Antib Immunodiagn Immunother. 2013;32(2):73-80.

49. Watanabe $\mathrm{R}$, Hanaoka $\mathrm{H}$, Sato $\mathrm{K}$, et al. Photoimmunotherapy targeting prostate-specific membrane antigen: are antibody fragments as effective as antibodies? J Nucl Med. 2015;56(1):140-4. 
50. Fonseca MJ, Jagtenberg JC, Haisma HJ, Storm G. Liposome-mediated targeting of enzymes to cancer cells for site-specific activation of prodrugs: comparison with the corresponding antibody-enzyme conjugate. Pharm Res. 2003;20(3):423-8.

51. Boonstra MC, Van Driel PB, Van Willigen DM, et al. uPAR-targeted multimodal tracer for pre- and intraoperative imaging in cancer surgery. Oncotarget. 2015;6(16):14260-73.

52. Ghosh SC, Ghosh P, Wilganowski N, et al. Multimodal chelation platform for near-infrared fluorescence/nuclear imaging. J Med Chem. 2013;56(2):406-16

Submit your next manuscript to BioMed Central and we will help you at every step:

- We accept pre-submission inquiries

- Our selector tool helps you to find the most relevant journal

- We provide round the clock customer support

- Convenient online submission

- Thorough peer review

- Inclusion in PubMed and all major indexing services

- Maximum visibility for your research

Submit your manuscript at www.biomedcentral.com/submit 